\title{
Mechanism overview and target mining of atherosclerosis: Endothelial cell injury in atherosclerosis is regulated by glycolysis (Review)
}

\author{
RUIYING WANG ${ }^{1-5}$, MIN WANG ${ }^{1-5}$, JINGXUE YE $^{1-5}$, GUIBO SUN $^{1-5}$ and XIAOBO SUN ${ }^{1-5}$ \\ ${ }^{1}$ Institute of Medicinal Plant Development, Peking Union Medical College and Chinese Academy of Medical Sciences; \\ ${ }^{2}$ Beijing Key Laboratory of Innovative Drug Discovery of Traditional Chinese Medicine (Natural Medicine) and \\ Translational Medicine, Institute of Medicinal Plant Development, Peking Union Medical College and \\ Chinese Academy of Medical Sciences; ${ }^{3}$ Key Laboratory of Bioactive Substances and Resources Utilization of \\ Chinese Herbal Medicine, Ministry of Education, Institute of Medicinal Plant Development, Peking Union Medical College \\ and Chinese Academy of Medical Sciences; ${ }^{4}$ Key Laboratory of Efficacy Evaluation of Chinese Medicine Against Glycolipid \\ Metabolic Disorders, State Administration of Traditional Chinese Medicine, Institute of Medicinal Plant Development, \\ Peking Union Medical College and Chinese Academy of Medical Sciences; ${ }^{5}$ Key Laboratory of New Drug Discovery Based \\ on Classic Chinese Medicine Prescription, Chinese Academy of Medical Sciences, Beijing 100193, P.R. China
}

Received August 14, 2020; Accepted November 5, 2020

DOI: $10.3892 /$ ijmm.2020.4798

\begin{abstract}
Atherosclerosis (AS) is a chronic disease with a complex pathology that may lead to several cardiovascular and cerebrovascular diseases; however, further research is necessary to fully elucidate its pathogenesis. The main risk factors for AS include lipid metabolism disorders, endothelial cell injury, inflammation and immune dysfunction, among which vascular endothelial cell damage is considered as the main trigger for AS occurrence and development. Endothelial cell damage leads to enhanced intimal permeability and leukocyte adhesion, promoting thrombus formation and accelerating disease progression. The function of endothelial cells is affected by glycolysis regulation, since $80 \%$ of ATP in these cells is produced via this pathway. Genes associated with AS and endothelial cell glycolysis, including AKT1, interleukin-6, vascular endothelial growth factor A, TP53, signal transducer and activator of transcription 3, SRC and mitogen-activated protein kinase 1, were screened. Through integrated analysis, these genes were found to play a key role in AS by regulating multiple signaling pathways associated with cell signal transduction, energy metabolism, immune function and thrombosis.
\end{abstract}

Correspondence to: Professor Guibo Sun or Professor Xiaobo Sun, Institute of Medicinal Plant Development, Peking Union Medical College and Chinese Academy of Medical Sciences, 151 Malianwa North Road, Haidian, Beijing 100193, P.R. China

E-mail: sunguibo@126.com

E-mail: sun_xiaobo163@163.com

Key words: endothelial injury, atherosclerosis, glycolysis, target, mechanism
In conclusion, endothelial cell injury in AS may be alleviated by glycolysis and is a potential clinical treatment strategy for AS.

\section{Contents}

1. Introduction

2. Factors implicated in AS

3. Vascular endothelial cells

4. Glycolysis

5. Prediction and analysis of targets associated with endothelial cell glycolysis in AS

6. Discussion

\section{Introduction}

Atherosclerosis (AS) is a chronic disease with complex etiology, which involves early local injury of the arterial intima, followed by lipid deposition, proliferation of the intimal fibrous tissue, local thickening of the intima and, ultimately, plaque formation (1). Vascular plaque-induced stenosis by AS may lead to insufficient arterial blood supply and cardiovascular diseases (2). The most serious complications caused by plaque rupture are myocardial infarction, cerebral ischemia, and ischemia of the surrounding tissue (3). The pathogenesis of AS has not been fully elucidated to date. Previous studies have demonstrated that AS is associated with lipid metabolism disorders, endothelial cell damage, inflammation and immune dysfunction, involving macrophages, endothelial cells, vascular smooth muscle cells and platelets $(4,5)$. In recent years, AS animal models mainly include mice, rabbits, miniature pigs, non-human primates and transgenic animals (6). AS is a vicious circle combining multiple factors and long-term 
effects. So, elucidating the underlying mechanism is crucial for the treatment and prevention of the disease.

Despite not having been fully elucidated, it is believed that lipid metabolism disorders, endothelial cell injury, inflammation and immune dysfunction are the most important factors implicated in the pathogenesis of AS $(5,7)$. Functional damage of endothelial cells is the initiating step in the early stage of AS (8). Endothelial cell damage in AS plaques leads to further plaque instability, rupture (9) and secondary thrombosis, thus accelerating disease progression and affirming the important role of endothelial cell integrity (10). Glycolysis, the most important energy source for endothelial cells, is used to quickly produce energy, enabling cells to respond to environmental changes (11). Intermediate metabolic products produced during glycolysis affect cell survival (12); therefore, glycolytic rates in endothelial cells play a key role in maintaining their homeostasis and reducing the risk for AS.

The aim of the present study was to review the pathogenesis of AS, the role of endothelial cell damage and glycolysis, and the role of associated target genes and the involved signalling pathways, in order to indicate new approaches to the research on AS pathogenesis and intervention methods, and aid in the development of novel treatments for AS.

\section{Factors implicated in AS}

Pathogenesis. The pathogenesis of AS is extremely complicated (Fig. 1). AS is currently considered to be the result of the interaction among various mechanisms, including lipid metabolism disorder, inflammatory cell infiltration, oxidative stress, immune dysfunction and vascular endothelial cell damage, the latter of which ultimately leads to plaque rupture and thrombosis, leading to serious cardiovascular and cerebrovascular diseases $(1,4,13)$.

Role of oxidative stress in AS. Oxidative stress is the initiating factor of AS inflammatory response, with reactive oxygen species (ROS) and oxidized low-density lipoprotein (Ox-LDL; formed by oxidative modification of LDL) being the main factors responsible for endothelial cell damage and for inducing the expression of pro-inflammatory factors in endothelial cells $(14,15)$. When endothelial cells are continuously exposed to external as well as endogenous oxidants, oxidative stress is likely to induce production of various biologically active substances, which may cause endothelial cell functional damage and apoptosis $(16,17)$. This process leads to the synthesis and release of inflammatory factors, further aggravating vascular inflammation (18). In addition, oxidative stress regulates the expression of vascular wall genes by acting on transcription factors of the vascular wall cells. For example, intracellular ROS directly oxidatively modify the transcription factor itself, thereby participating in the occurrence and development of AS (14).

Role of lipid metabolism disorder in AS. Excessive blood lipid levels are the main cause of AS. In the hyperlipidemic state, the elevated plasma LDL cholesterol (LDL-C) is deposited on the vascular intima and enters macrophages via membrane receptors (19). In addition, LDL also undergoes oxidative modification to form Ox-LDL, leading to changes in endothelial cell function, and causing increased permeability and lipid deposition in the inner membrane (20). Ox-LDL exhibits strong affinity for scavenger receptors found on mononuclear macrophages, leading to its quick internalisation (21). However, Ox-LDL is toxic for macrophages, causing them to become activated, rapidly proliferate, aggregate and degenerate (22). Finally, the macrophages undergo apoptosis and become foam cells, which then aggregate to form AS lipid plaques. Moreover, Ox-LDL binds to vascular endothelial cells through lectin-like oxidized LDL receptor-1 to disrupt intracellular signaling and cause endothelial cell dysfunction (23). Ox-LDL can also promote the continuous proliferation of vascular smooth muscle cells and their outward migration to form plaques on the inner wall of blood vessels.

Role of endothelial cell injury in AS. Disruption of endothelial cell morphology and function leads to vascular barrier function impairment, as well as to changes in the intimal integrity and permeability (24). The apoptosis and shedding of endothelial cells promote the adhesion and aggregation of platelets from the blood (25). Dysfunctional endothelial cells, macrophages and platelets secrete a variety of growth factors and vasoactive substances, stimulating the continuous proliferation of smooth muscle cells in the media, and enter the intima, while also causing contractions of the vascular wall (26). As a result, the fatty plaques increase in size while the lumen becomes progressively narrowed, promoting the formation of AS lesions (8).

Role of inflammation in AS. AS involves not only lipid deposition in blood vessel walls, but also chronic inflammation (27). Oxidative stress persists throughout AS. AS has been proven to be a chronic inflammatory disease initiated in the arterial wall, mainly driven by modification of endogenous structures and dysfunction of the vascular endothelium (28). Certain lipids act as signaling molecules, and bind to cell receptors to activate the expression of specific genes and produce a number of pro-inflammatory cytokines (19). This leads to an increase in the numbers of inflammatory cells, including macrophages, increased phagocytosis of Ox-LDL and production of pro-inflammatory factors, such as tumor necrosis factor (TNF)- $\alpha$, interleukin (IL)-1 and IL-8 (29). These factors further aggravate the pathology of AS.

Role of immune dysfunction in AS. AS is also an autoimmune disease, stimulated by accumulated lipoproteins, as well as specific $\mathrm{T}$ lymphocytes and their antibodies, in the blood vessel wall (30). It was previously demonstrated that $\mathrm{T}$ lymphocytes infiltrate the aorta, where they accumulate and express restricted T-cell receptors, thereby promoting AS through immune regulation (13). In addition, the cells of innate immune response, such as monocytes and neutrophils, play an important role in the occurrence and development of AS (31). Upon immune function impairment, these cells disrupt cytokine production (27). Thus, the expression of anti-inflammatory factors decreases and the expression of pro-inflammatory factors increases, ultimately promoting AS development (29).

Role of microRNAs (miRNA) of the circulatory system in AS. miRNAs are found in non-coding regions of the genome and 


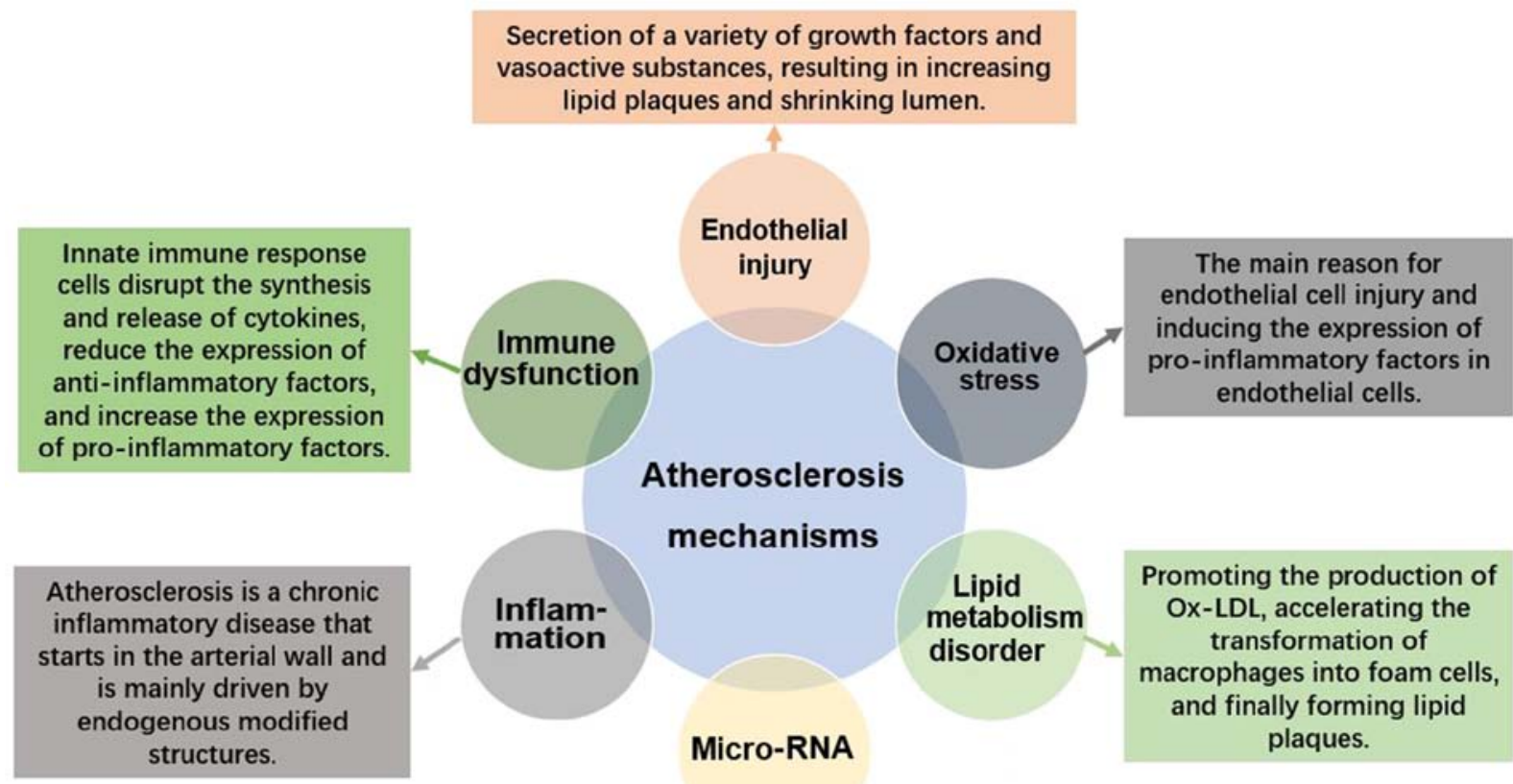

Participating in the regulation of inflammatory cells, vascular endothelial cells and monocytes in all aspects of the occurrence and development of AS.

Figure 1. Overall diagram of atherosclerosis pathogenesis. (AS) pathogenesis is generally considered to be the result of the interaction among various mechanisms including lipid metabolism disorder, inflammatory cell infiltration, oxidative stress, immune dysfunction, and vascular endothelial cell damage, the latter of which ultimately leads to plaque rupture and thrombosis, causing serious cardiovascular and cerebrovascular diseases. Besides, miRNAs are involved in inflammatory cell regulation and thus other aspects of AS pathogenesis. AS, atherosclerosis; Ox-LDL, oxidized low-density lipoprotein.

play important roles in gene transcription, post-transcriptional processing, cell proliferation, cell differentiation, cell apoptosis, ontogeny, heredity and epigenetics $(21,23)$. It has been recently discovered that miRNAs are involved in inflammatory cell regulation and, thus, all aspects of AS pathology, including vascular endothelial cell and monocyte development, differentiation and function (32). Studies have shown that miR-143 can affect the formation of AS plaques by inhibiting endothelial cell glycolysis, while miR-33 is closely associated with macrophage metabolism $(33,34)$.

Drug intervention for AS. The aim of currently available anti-AS treatments, which mainly include statins, antithrombotic drugs and surgical intervention, is to reduce serum LDL levels (35). Statins are methylglutaryl-CoA reductase inhibitors that have been found to be effective at halting disease progression and reducing the incidence of cardiovascular and cerebrovascular complications. By inhibiting methylglutaryl-CoA reductase, statins lower total cholesterol and LDL-C, increase HDL-C, activate nitric oxide (NO) synthase, increase endothelial NO levels, and prevent NO decrease caused by Ox-LDL (36). Probucol, a strong synthetic antioxidant, is a symmetrical di-tert-butylphenol structure that is easily oxidized, thus reducing free oxygen radicals and diminishing their oxidizing capacity (37).

The irreversible antioxidant effect of probucol is attributed to its strong affinity for LDL-C, thus inhibiting LDL-C and reducing the formation of Ox-LDL. Anti-platelet aggregation drugs may also exert an anti-inflammatory effect on the injured vascular intima (38).
The combination of statins and antioxidants may prevent thrombosis and AS plaque formation (39). In addition, niacin, a broad-spectrum lipid-modulating drug, acts by inhibiting the expression of serum adhesion molecules and inflammatory cytokines (40). The anti-inflammatory effect of niacin is achieved by downregulating the $\mathrm{NF}-\kappa \mathrm{B}$ signaling pathway.

In addition to Western medicine, Traditional Chinese Medicine (TCM) has also achieved marked benefits in the treatment of AS. In TCM, AS is commonly categorised as 'blood stasis' and 'phlegm turbidity' (41). Modern TCM theories mostly attribute the pathogenesis of AS to phlegm, toxins and stasis. TCM arrests AS progression by activating blood and dredging collaterals, inhibiting inflammation and plaque formation, and stabilizing plaques $(42,43)$. Commonly used TCM agents include Chaihu Shugan San, Yiqi Yangyin Recipe, Danhuang Tongmai Capsule and Simiao Yongan Decoction (44-46). From a macro perspective, TCM is guided by Chinese medicine principles, and the treatment is performed holistically. In recent years, TCM has been found to add unique advantages to the treatment of complex diseases, such as AS.

\section{Vascular endothelial cells}

Physiological functions of vascular endothelial cells. The inner vascular endothelium in the heart, blood and lymphatic vessels is composed of squamous epithelial cells, and is important for maintaining the complete structure and function of the blood vessel wall (47). Endothelial cells serve not only 
as a mechanical barrier, but also as receptors and endocrine organs, as they are capable of synthesizing and releasing a variety of endothelial-derived vasoactive factors (9). When damaged, vascular endothelial cells secrete a variety of molecules to promote leukocyte adhesion. They also secrete endothelium-dependent factors, such as endothelin, angiotensin, NO and prostacyclin, regulating vasoconstriction and vasodilation (48). Endothelium-dependent dilation factors may also inhibit platelet activation and aggregation. Plasma plasminogen activator synthesized by vascular endothelial cells has high affinity for fibrin and can activate plasminogen to dissolve thrombi (24). Under normal conditions, these cells reduce inflammation by regulating the release of various cytokines and inflammatory factors, such as intercellular adhesion molecule-1, vascular cell adhesion molecule-1 and monocyte chemokines (49). However, the injured endothelium promotes a series of inflammatory reactions, causing endothelial cell dysfunction and favouring AS development.

Mechanism underlying the development of AS caused by vascular endothelial cell injury. Vascular endothelial cell function is closely associated with AS, which is a chronic inflammatory response of the arterial wall to endothelial cell injury (8). Vascular endothelial cell injury and functional damage serve as the initiating factor. The dysfunction and morphological damage of endothelial cells may manifest as a variety of endothelial abnormalities, causing leukocyte adhesion, vasoconstriction, platelet activation, oxidative stress and inflammation, followed by thrombus formation which, in turn, promotes the formation of AS plaques $(24,25,50,51)$. Endothelial progenitor cells participate in reendothelialization and angiogenesis, and may play a pivotal role in the occurrence and development of AS (52).

Increased intimal permeability. Following endothelial cell injury, lipids accumulate in the blood vessels, resulting in increased permeability of the vascular intima. This causes monocytes and phagocytes to be released into the bloodstream, increasing the adhesion to endothelial cells (50). LDL is then oxidized to the cytotoxic Ox-LDL, which is damaging to endothelial and smooth muscle cells. Macrophage Ox-LDL uptake occurs through scavenger receptors, accelerating their transformation into foam cells that eventually form the AS plaques (47).

Increased leukocyte adhesion. Leukocytes, particularly monocytes, adhere to the endothelium through intercellular adhesion molecules, leukocyte adhesion molecules, TNF- $\alpha$ and vascular cell adhesion molecules. Subsequently, guided by chemokines, they migrate to the intima and differentiate into macrophages (53). Macrophages secrete a variety of inflammatory factors, such as TNF- $\alpha$, IL- 1 and IL- 6 , to promote the formation of AS plaques. In addition, damage to endothelial cells leads to increased expression of adhesion molecules, while also increasing the susceptibility of endothelial cells to inflammatory stimuli. This again increases the expression of adhesion molecules, promoting the adhesion of monocytes to endothelial cells and triggering AS development (34).

Promoting thrombosis. Stimulation or damage of endothelial cells causes a decrease in their antithrombotic properties, which promotes thrombus formation (25). Among other factors, increased secretion of the von Willebrand factor and thromboxane mediate platelet aggregation and adhesion to the endothelium to promote thrombosis, while plasminogen activator inhibitors inhibit thrombolysis (5). Thus, the microthrombi formed on the endothelial inner membrane are difficult to dissolve, contributing to the formation of plaques (8).

Regulation of endothelial progenitor cells. Endothelial progenitor cells, a type of precursor cell with a high proliferation potential, are a heterogeneous cell population with multiple origins and different phenotypes, capable of producing endothelial cells (54). Endothelial progenitor cells can selectively be recruited to damaged or ischemic areas by stress and inflammatory stimuli to form new blood vessels through differentiation and proliferation, without relying on the original vasculature (55). Inflammation is the pathophysiological basis of various cardiovascular diseases. Pro-inflammatory cytokines stimulate the expression of adhesion molecules on the surface of endothelial cells and promote the onset of AS (56). Inflammation can stimulate the release of endothelial progenitor cells from the bone marrow into peripheral blood, thus promoting tissue repair (57). Therefore, the occurrence of AS is associated with endothelial progenitor cells, which are involved in repair and intimal hyperplasia following endothelial cell injury, which also promote the formation and stability of AS plaques (52).

\section{Glycolysis}

It was previously demonstrated that $85 \%$ of ATP in endothelial cells is produced by glycolysis, of which $\sim 60 \%$ is used for homeostatic maintenance and $40 \%$ for proliferation (11). The high glycolytic properties of endothelial cells result from their low content of mitochondria, which comprise only $5 \%$ of the cell. Although endothelial cells reside in a high-oxygen environment, they consume little oxygen and can, therefore, deliver oxygen to nearby tissues (58). Endothelial cells use glycolysis to quickly produce energy, which helps them adapt to changes in their environment. More metabolic intermediate products are produced through glycolysis, affecting cell survival (59). Compared with other cells, resting endothelial cells have a high-efficiency glycolysis rate. When cells undergo migration or proliferation, their glycolysis rates double (60). Glycolysis is required to meet the energy demands of blood vessel sprouting, since the apical cells at the front must continuously migrate forward to form filamentous or laminar pseudopodia, while stem cells at the back increase their proliferation rate and form a lumen (61). Endothelial cell dysfunction caused by Ox-LDL and other factors is the main cause of AS. It was previously demonstrated that excessive activation of glycolysis is a key factor leading to endothelial cell dysfunction and proliferation (12). Maintaining the metabolic balance of endothelial cells by inhibiting glycolysis to reduce dysfunction and inflammation may represent a novel treatment strategy for AS (11). The rate-limiting enzymes of glycolysis may be a bridge to elucidating the association between endothelial cell injury and AS.

Under physiological conditions, cells metabolize glucose mainly through oxidative phosphorylation, while 
the glycolytic pathway is activated only under hypoxic conditions (62). The glycolytic pathway comprises a series of enzymatic reactions in which glucose from tissues is degraded (63). Glucose is first transported into the cell via glucose transporters (GLUTs), and is then phosphorylated by hexokinase (HK) into glucose-6-phosphate, which cannot penetrate the cell membrane (64). Glucose-6-phosphate is then converted into pyruvate by hexose phosphate isomerase and phosphofructokinase (PFK) (65). The pyruvate produced through glycolysis may directly enter the tricarboxylic acid cycle or be converted into lactic acid by lactate dehydrogenase (LDHA) (66). The lactic acid is then transported outside the cell via the monocarboxylic acid transporter $1 / 4$ (67). The ATP produced by this process provides energy for the cell.

Regulation of HK in endothelial glycolysis. HK is the first rate-limiting enzyme in the glycolytic pathway. This enzyme catalyses the conversion of glucose to glucose-6-phosphate, and produces ATP through oxidative phosphorylation or glycolysis (68). It was previously demonstrated that the AKT/mTOR signalling pathway is associated with HK production. High HK expression in endothelial cells facilitates efficient glycolysis and promotes rapid cell proliferation (69). In addition, HK can bind to voltage-dependent anion channels in the outer mitochondrial membrane to prevent binding of the pro-apoptotic protein Bax. This prevents the release of cytochrome $c$ from the mitochondria, thereby exerting an anti-apoptotic effect (70). Therefore, HK not only promotes cell proliferation, but also inhibits cell apoptosis.

Regulation of PFK in endothelial glycolysis. PFK, the second glycolytic rate-limiting enzyme in the glycolytic pathway, catalyses the conversion of fructose 6-phosphate to fructose 1,6-diphosphate. The expression of PFK is regulated by a number of factors, such as c-Src activation and hypoxia-inducible factor (HIF)-1 $\alpha$, promoting PFK2 expression (71). TP53-induced glycolysis and apoptosis regulator (TIGAR) may reduce $\mathrm{PFK}$ expression and inhibit glycolysis. The kinase activity of PFKFB3, a subtype of PFK2, in vascular endothelial cells, is affected by the RAS and AMP-activated protein kinase (AMPK) signalling pathways (72). In addition, PFKFB3 can also promote endothelial cell inflammation through TNF- $\alpha$, thereby promoting AS development (73).

Regulation of PK in endothelial glycolysis. PK, the third rate-limiting enzyme in the glycolytic pathway, specifically catalyses the conversion of phosphoenolpyruvate to pyruvate. This irreversible reaction is a crucial regulatory step in the glycolytic pathway. PKM2 is the only PK subtype that can switch between high-activity (tetramer) and low-activity (dimer) forms. Following phosphorylation, PKM2 is converted to a dimer, promoting the upstream glycolytic products of $\mathrm{PK}$ to enter the biosynthetic pathway (74). In addition, the expression of PKM2 is differentially regulated by the PI3K/AKT signalling pathway (75). PKM2-regulated glycolysis contributes to the proliferation and migration of vascular smooth muscle cells, and is positively correlated with AS development (12).

Regulation of LDHA in endothelial glycolysis. LDHA, the fourth rate-limiting enzyme in the glycolytic pathway, catalyses the production of lactic acid from pyruvate. This enzyme enables recycling of pyruvate and reduced nicotinamide purine dinucleotide, and plays a key role in promoting efficient cell glycolysis (76). SRC can also phosphorylate LDHA and promote conversion enzyme activity. HIF- $1 \alpha$ is the upstream regulator of LDHA, and both HIF-1 $\alpha$ and LDHA stimulate the inflammatory response (77).

\section{Prediction and analysis of targets associated with endothelial cell glycolysis in AS}

Target prediction. Although endothelial cell glycolysis plays an important role in AS, related research is scarce. Therefore, in the present study, the term 'NCBI-gene' was used to search for genes associated with endothelial cell glycolysis in AS, and 76 genes were identified (Table I). The Comparative Toxicogenomics Database (CTD) is a database used to describe the association between chemical substances, genes and human diseases. CTD was used to conduct Kyoto Encyclopedia of Genes and Genomes pathway enrichment analysis (Table II); the pathways included in the analysis were the immune system pathway, the signal transduction pathway, pathways in cancer, the PI3K/AKT, IL-4, IL-13, HIF-1, NGF and FoxO signalling pathways, the platelet activation, signalling and aggregation pathway, as well as the fluid shear stress and AS pathways.

The screened genes play important regulatory roles in multiple signalling pathways, indicating that such interactions should also be present among their corresponding proteins. The STRING database, used to study the protein interaction networks, helps to mine core regulatory genes. Based on that, a protein-protein interaction network was built and Cytoscape 3.2.1 software (https://cytoscape. org/download_old_versions.html) was used to visualize it (Fig. 2). The node size in Fig. 1 reflects its importance in the network. CytoHubba is a network analysis plug-in specifically used to identify key nodes in the Cytoscape software, providing a variety of key node identification algorithms, which focus on different network topology characteristics. To make the identification of key targets as accurate as possible, the top 10 key targets were identified based on two algorithms, namely Matthews correlation coefficient (MCC) and Stress (Fig. 3). Next, the intersection of the two results was considered as the key targets for AS endothelial glycolysis, including AKT1, IL-6, vascular endothelial growth factor (VEGF)A, TP53, signal transducer and activator of transcription 3 (STAT3), SRC and mitogen-activated protein kinase (MAPK)1.

Target analysis. All 7 predicted targets are factors proven to promote or inhibit the process of AS. AKT1 and TP53 can directly regulate glycolysis, thereby affecting AS. In addition to the regulation of glycolysis, IL- 6 can also regulate AS through inflammation; VEGFA, STAT3 and SRC are also closely associated with immune system dysfunction. The regulatory effect of MAPK1 on AS is mainly mediated through immune response, but the association between glycolysis and MAPK1 has not been explored. The available research on these 7 targets is currently not sufficient, and further experiments are required to verify the results. The purpose of the present review was also to provide a direction for future AS studies. 
Table I. Gene information on atherosclerosis and endothelial cell glycolysis.

\begin{tabular}{|c|c|c|c|c|c|}
\hline No. & Gene name & Full name & Chromosome & Location & ID \\
\hline 1 & VEGFA & Vascular endothelial growth factor A & 6 & $6 \mathrm{p} 21.1$ & 7422 \\
\hline 2 & CXCL8 & $\mathrm{C}-\mathrm{X}-\mathrm{C}$ motif chemokine ligand 8 & 4 & $4 q 13.3$ & 3576 \\
\hline 3 & PPARG & Peroxisome proliferator activated receptor gamma & 3 & $3 \mathrm{p} 25.2$ & 5468 \\
\hline 4 & IL33 & Interleukin 33 & 9 & $9 \mathrm{p} 24.1$ & 90865 \\
\hline 5 & IL6 & Interleukin 6 & 7 & $7 \mathrm{p} 15.3$ & 3569 \\
\hline 6 & HIF1A & Hypoxia-inducible factor 1 subunit alpha & 14 & $14 q 23.2$ & 3091 \\
\hline 7 & TP53 & Tumor protein p53 & 17 & $17 \mathrm{p} 13.1$ & 7157 \\
\hline 8 & KDR & Kinase insert domain receptor & 4 & $4 q 12$ & 3791 \\
\hline 9 & TGFB1 & Transforming growth factor beta 1 & 19 & $19 q 13.2$ & 7040 \\
\hline 10 & $\operatorname{miR} 21$ & MicroRNA 21 & 17 & $17 q 23.1$ & 406991 \\
\hline 11 & CDKN2A & Cyclin-dependent kinase inhibitor 2A & 9 & $9 \mathrm{p} 21.3$ & 1029 \\
\hline 12 & IL1B & Interleukin 1 beta & 2 & $2 q 14.1$ & 3553 \\
\hline 13 & AKT1 & AKT serine/threonine kinase 1 & 14 & $14 q 32.33$ & 207 \\
\hline 14 & BCL2 & BCL2 apoptosis regulator & 18 & $18 \mathrm{q} 21.33$ & 596 \\
\hline 15 & SIRT1 & Sirtuin 1 & 10 & $10 \mathrm{q} 21.3$ & 23411 \\
\hline 16 & STAT3 & Signal transducer and activator of transcription 3 & 17 & $17 \mathrm{q} 21.2$ & 6774 \\
\hline 17 & TLR4 & Toll-like receptor 4 & 9 & $9 q 33.1$ & 7099 \\
\hline 18 & PTGS2 & Prostaglandin-endoperoxide synthase 2 & 1 & $1 \mathrm{q} 31.1$ & 5743 \\
\hline 19 & ADIPOQ & Adiponectin C1Q and collagen domain containing & 3 & $3 q 27.3$ & 9370 \\
\hline 20 & NFE2L2 & Nuclear factor erythroid 2 like 2 & 2 & $2 q 31.2$ & 4780 \\
\hline 21 & NOTCH1 & Notch receptor 1 & 9 & $9 q 34.3$ & 4851 \\
\hline 22 & MTOR & Mechanistic target of rapamycin kinase & 1 & $1 \mathrm{p} 36.22$ & 2475 \\
\hline 23 & CTNNB1 & Catenin beta 1 & 3 & $3 \mathrm{p} 22.1$ & 1499 \\
\hline 24 & PTEN & Phosphatase and tensin homolog & 10 & $10 \mathrm{q} 23.31$ & 5728 \\
\hline 25 & LEP & Leptin & 7 & 7q32.1 & 3952 \\
\hline 26 & ESR1 & Estrogen receptor 1 & 6 & $6 \mathrm{q} 25.1-\mathrm{q} 25.2$ & 2099 \\
\hline 27 & SOD1 & Superoxide dismutase 1 & 21 & $21 \mathrm{q} 22.11$ & 6647 \\
\hline 28 & MAPK1 & Mitogen-activated protein kinase 1 & 22 & $22 q 11.22$ & 5594 \\
\hline 29 & RELA & RELA proto-oncogene NF-kB subunit & 11 & $11 q 13.1$ & 5970 \\
\hline 30 & $\operatorname{miR} 34 \mathrm{~A}$ & MicroRNA 34a & 1 & $1 \mathrm{p} 36.22$ & 407040 \\
\hline 31 & IFNG & Interferon gamma & 12 & $12 q 15$ & 3458 \\
\hline 32 & AGTR1 & Angiotensin II receptor type 1 & 3 & $3 q 24$ & 185 \\
\hline 33 & $\mathrm{ABCB} 1$ & ATP-binding cassette subfamily B member 1 & 7 & $7 q 21.12$ & 5243 \\
\hline 34 & CD44 & CD44 molecule (Indian blood group) & 11 & $11 \mathrm{p} 13$ & 960 \\
\hline 35 & STAT1 & Signal transducer and activator of transcription 1 & 2 & $2 q 32.2$ & 6772 \\
\hline 36 & HGF & Hepatocyte growth factor & 7 & $7 q 21.11$ & 3082 \\
\hline 37 & NAMPT & Nicotinamide phosphoribosyl transferase & 7 & $7 q 22.3$ & 10135 \\
\hline 38 & CYBB & Cytochrome b- 245 beta chain & $\mathrm{X}$ & Xp21.1-p11.4 & 1536 \\
\hline 39 & $\mathrm{KL}$ & Klotho & 13 & $13 q 13.1$ & 9365 \\
\hline 40 & $\mathrm{SRC}$ & SRC proto-oncogene non-receptor tyrosine kinase & 20 & $20 q 11.23$ & 6714 \\
\hline 41 & PPARA & Peroxisome proliferator activated receptor alpha & 22 & $22 \mathrm{q} 13.31$ & 5465 \\
\hline 42 & BSG & Basigin (Ok blood group) & 19 & $19 \mathrm{p} 13.3$ & 682 \\
\hline 43 & TXN & Thioredoxin & 9 & $9 q 31.3$ & 7295 \\
\hline 44 & NOS2 & Nitric oxide synthase 2 & 17 & $17 q 11.2$ & 4843 \\
\hline 45 & FOXO3 & Forkhead box $\mathrm{O} 3$ & 6 & $6 q 21$ & 2309 \\
\hline 46 & PRKCE & Protein kinase $\mathrm{C}$ epsilon & 2 & $2 \mathrm{p} 21$ & 5581 \\
\hline 47 & NR4A1 & Nuclear receptor subfamily 4 group A member 1 & 12 & $12 q 13.13$ & 3164 \\
\hline 48 & NOX4 & NADPH oxidase 4 & 11 & $11 q 14.3$ & 50507 \\
\hline 49 & PRKAA1 & Protein kinase AMP-activated catalytic subunit alpha 1 & 5 & $5 \mathrm{p} 13.1$ & 5562 \\
\hline 50 & $\operatorname{miR} 210$ & MicroRNA 210 & 11 & $11 \mathrm{p} 15.5$ & 406992 \\
\hline 51 & MALAT1 & Metastasis-associated lung adenocarcinoma transcript 1 & 11 & $11 \mathrm{q} 13.1$ & 378938 \\
\hline 52 & IGF1R & Insulin-like growth factor 1 receptor & 15 & $15 \mathrm{q} 26.3$ & 3480 \\
\hline
\end{tabular}


Table I. Continued.

\begin{tabular}{lllrrr}
\hline No. & Gene name & \multicolumn{1}{c}{ Full name } & Chromosome & Location & ID \\
\hline 53 & AHR & Aryl hydrocarbon receptor & 7 & $7 \mathrm{p} 21.1$ & 196 \\
54 & CD36 & CD36 molecule & 7 & $7 \mathrm{q} 21.11$ & 948 \\
55 & EZH2 & Enhancer of zeste 2 polycomb repressive complex 2 subunit & 7 & $7 \mathrm{q} 36.1$ & 2146 \\
56 & SIRT6 & Sirtuin 6 & 19 & $19 \mathrm{p} 13.3$ & 51548 \\
57 & IKBKB & Inhibitor of nuclear factor kappa B kinase subunit beta & 8 & $8 \mathrm{p} 11.21$ & 3551 \\
58 & miR122 & MicroRNA 122 & 18 & $18 \mathrm{q} 21.31$ & 406906 \\
59 & UCP2 & Uncoupling protein 2 & 11 & $11 \mathrm{q} 13.4$ & 7351 \\
60 & ENO1 & Enolase 1 & 1 & $1 \mathrm{p} 36.23$ & 2023 \\
61 & PPARGC1A & PPARG coactivator 1 alpha & 4 & $4 \mathrm{p} 15.2$ & 10891 \\
62 & SNAI1 & Snail family transcriptional repressor 1 & 20 & $20 \mathrm{q} 13.13$ & 6615 \\
63 & PPBP & Pro-platelet basic protein & 4 & $4 \mathrm{q} 13.3$ & 5473 \\
64 & DICER1 & Dicer 1 ribonuclease III & 14 & $14 \mathrm{q} 32.13$ & 23405 \\
65 & TXNIP & Thioredoxin-interacting protein & 1 & $1 \mathrm{q} 21.1$ & 10628 \\
66 & IL22 & Interleukin 22 & 12 & $12 \mathrm{q} 15$ & 50616 \\
67 & TNFSF13B & TNF superfamily member 13b & 13 & $13 \mathrm{q} 33.3$ & 10673 \\
68 & SREBF2 & Sterol regulatory element-binding transcription factor 2 & 22 & $22 \mathrm{q} 13.2$ & 6721 \\
69 & miR497 & MicroRNA 497 & 17 & $17 \mathrm{p} 13.1$ & 574456 \\
70 & miR206 & MicroRNA 206 & 6 & $6 \mathrm{p} 12.2$ & 406989 \\
71 & TUG1 & Taurine upregulated 1 & 22 & $22 \mathrm{q} 12.2$ & 55000 \\
72 & miR135B & MicroRNA 135b & 1 & $1 \mathrm{q} 32.1$ & 442891 \\
73 & miR142 & MicroRNA 142 & 17 & $17 \mathrm{q} 22$ & 406934 \\
74 & miR135A1 & MicroRNA 135a-1 & 3 & $3 \mathrm{p} 21.2$ & 406925 \\
75 & miR148B & MicroRNA 148b & 12 & $12 \mathrm{q} 13.13$ & 442892 \\
76 & miR30C1 & MicroRNA 30c-1 & 1 & $1 \mathrm{p} 34.2$ & 407031 \\
\hline
\end{tabular}

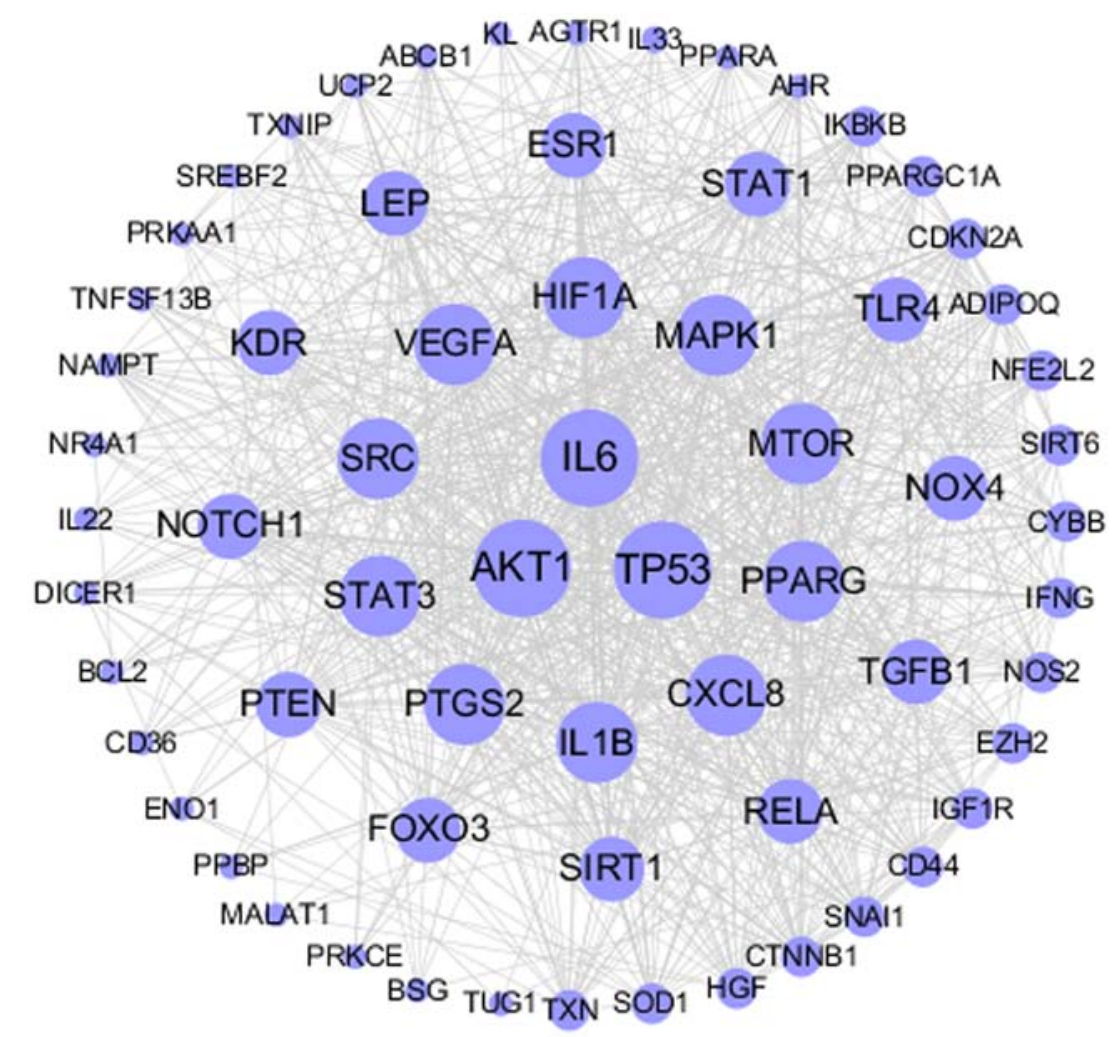

Figure 2. Protein interaction network of endothelial glycolysis and AS. The round nodes represent genes associated with endothelial glycolysis and AS. The size of the node reflects the importance of the node in the network to a certain extent: The larger the node, the greater the connectivity of the node in the network, and vice versa. Gray lines represent the interaction between two nodes. AS, atherosclerosis. 
Table II. Potential pathways of atherosclerosis and endothelial cell glycolysis.

\begin{tabular}{lll}
\hline Pathway & No. & \multicolumn{1}{c}{ Genes } \\
\hline Immune system & 36 & AKT1, BCL2, CD36, CD44, CTNNB1, CXCL8, CYBB, FOXO3, HGF, \\
& & HIF1A, IFNG, IKBKB, IL1B, IL22, IL33, IL6, KL, MAPK1, MTOR, NOS2, \\
& & NR4A1, PPBP, PRKCE, PTEN, PTGS2, RELA, SRC, STAT1, STAT3, TGFB1, \\
& & TLR4, TNFSF13B, TP53, TXN, TXNIP, VEGFA
\end{tabular}

Signal transduction

30

Pathways in cancer

PI3K-AKT signalling pathway

Cellular responses to stress

Interleukin-4 and 13 signalling

HIF-1 signalling pathway

Platelet activation, signaling and

aggregation

NGF signalling via TRKA from

the plasma membrane

Fluid shear stress and

atherosclerosis

FoxO signalling pathway

PI3K/AKT activation

10

LAT2/NTAL/LAB on calcium mobilization
AGTR1, AKT1, CTNNB1, CXCL8, CYBB, ESR1, FOXO3, HGF, HIF1A, IGF1R, IKBKB, IL6, KDR, KL, LEP, MAPK1, MTOR, NOTCH1, NR4A1, PPBP, PRKAA1, PRKCE, PTEN, RELA, SRC, STAT1, STAT3, TGFB1, TP53, VEGFA

AGTR1, AKT1, BCL2, CDKN2A, CTNNB1, CXCL8, HGF, HIF1A, IGF1R, IKBKB, IL6, MAPK1, MTOR, NOS2, PPARG, PTEN, PTGS2, RELA, STAT1, STAT3, TGFB1, TP53, VEGFA

AKT1, BCL2, FOXO3, HGF, IGF1R, IKBKB, IL6, KDR, MAPK1, MTOR, NR4A1, PRKAA1, PTEN, RELA, TLR4, TP53, VEGFA

CDKN2A, CXCL8, CYBB, EZH2, HIF1A, IL6, MAPK1, MTOR, NOX4, PRKAA1, RELA, SIRT1, SOD1, STAT3, TP53, TXN, VEGFA

AKT1, BCL2, CD36, CXCL8, FOXO3, HGF, HIF1A, IL1B, IL6, NOS2, PTGS2, STAT1, STAT3, TGFB1, TP53, VEGFA

5 AKT1, BCL2, CYBB, ENO1, HIF1A, IFNG, IGF1R, IL6, MAPK1, MTOR, NOS2, RELA, STAT3, TLR4, VEGFA

AKT1, CD36, HGF, MAPK1, PPBP, PRKCE, SOD1, SRC, TGFB1, VEGFA

AKT1, FOXO3, HGF, KL, MAPK1, MTOR, NR4A1, PRKCE, PTEN, SRC, STAT3, TP53

15 AKT1, BCL2, CTNNB1, CYBB, IFNG, IKBKB, IL1B, KDR, NFE2L2, PRKAA1, RELA, SRC, TP53, TXN, VEGFA

11 AKT1, FOXO3, IGF1R, IKBKB, IL6, MAPK1, PRKAA1, PTEN, SIRT1, STAT3, TGFB1

10 AKT1, FOXO3, HGF, KL, MAPK1, MTOR, NR4A1, PTEN, SRC, TP53
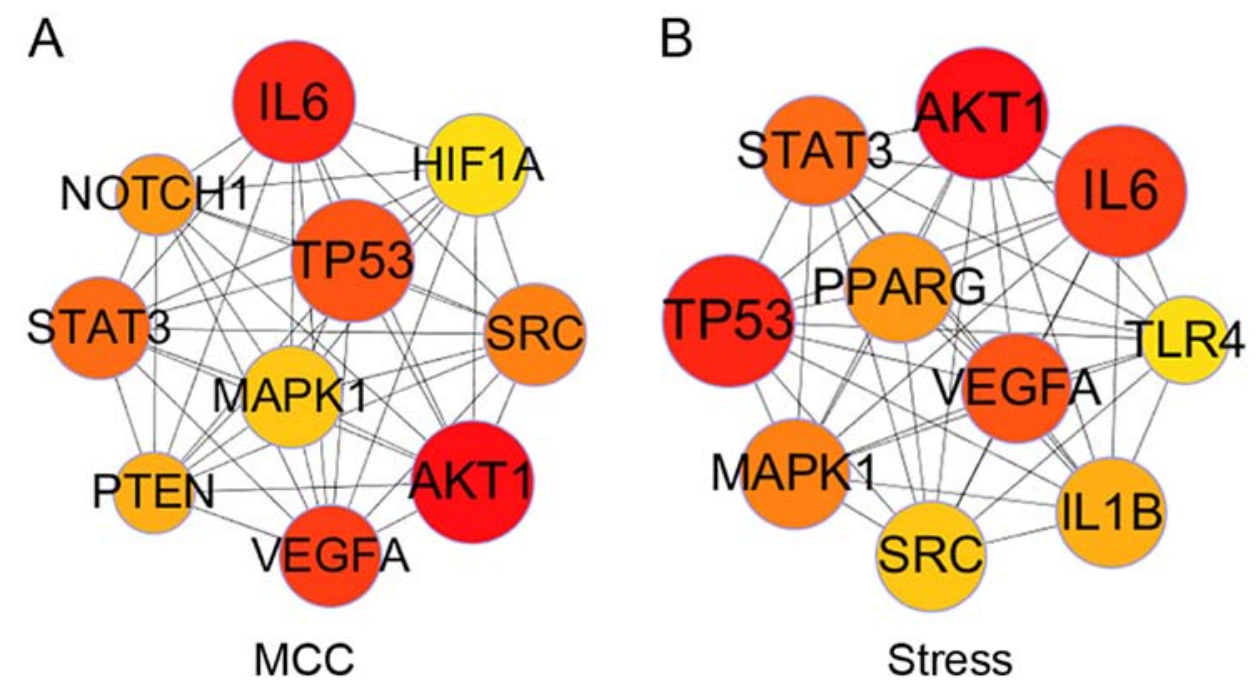

Figure 3. Identification of key protein targets. The top 10 key targets were identified based on the two algorithms, namely (A) MCC and (B) Stress, in Cytoscape software. The size and color of the nodes reflect the importance of the target (the larger the size and the darker the color, the more important the target). MCC, Matthews correlation coefficient. 
Evaluation of AKT1 involvement in endothelial cell glycolysis in AS. AKT, including AKT1, AKT2 and AKT3, play an important role in cell proliferation, survival, metabolism and migration (78). AKT can activate mTOR, another major regulatory gene of cell metabolism, thereby promoting the expression of HIF-1 $\alpha$. HIF-1 $\alpha$ then regulates the expression of glycolysis-related proteins, such as GLUTs, PK, HK2 and LDHA (79). These pathways promote glucose uptake and lactic acid production. In addition, endothelial glycolysis can be regulated by the FKBP51/AKT1 and AKT1/GSK/3 $\beta$ signalling pathways $(80,81)$. Therefore, as regards energy metabolism, AKT1 may be a key target in AS.

Evaluation of IL-6 involvement in endothelial cell glycolysis in AS. IL-6 is an important pro-inflammatory cytokine (82). Chronic inflammation is also considered as part of AS pathology, suggesting a possible role for IL-6 (83). It has been shown that IL-6 affects AS development through the acute phase response, and exerts an effect on insulin resistance (84), while it also promotes glycolysis through PFKFB3 (85). The IL-6/STAT3 pathway is also a pathway involved in glycolysis regulation (86).

Evaluation of VEGFA involvement in endothelial cell glycolysis in AS. VEGFA promotes angiogenesis and regulates endothelial cell proliferation, macrophage infiltration and foam cell formation through signal transduction (87); therefore, it plays a key regulatory role in the formation and stabilization of AS plaques (88). In addition, it was previously demonstrated that endothelial glycolysis may be promoted by enhancing VEGFA expression, making it an important contributor to AS development and endothelial glycolysis (89). Therefore, VEGFA is an important target in AS and endothelial glycolysis.

Evaluation of TP53 involvement in endothelial cell glycolysis in AS. TP53, also referred to as 'the guardian of the genome', maintains gene stability. As it is mutated in $>50 \%$ of tumour cells, TP53 is used for cancer prediction and risk assessment (90). In addition, TP53 regulates energy metabolism through the AKT/mTOR and $\mathrm{NF}-\kappa \mathrm{B}$ signalling pathways, and it inhibits glycolysis by downregulating the expression of rate-limiting enzymes and activating TP53-induced glycolysis and TIGAR $(91,92)$. It was previously demonstrated that endothelial autophagy is inhibited in advanced AS by regulating mTOR and TIGAR (93). However, there is insufficient research on AS endothelial glycolysis, and the TP53 regulatory effects in AS require further investigation.

Evaluation of STAT3 involvement in endothelial cell glycolysis in AS. STAT3, a transcription factor, regulates cell growth, apoptosis and inflammation, and plays an important role in cancer, AS, as well as cardiovascular and cerebrovascular diseases (94). STAT3 is also strongly associated with endothelial cell dysfunction, macrophage polarization and inflammatory responses, thereby promoting AS development (95). In addition, it can enhance cell metabolism through HK2 upregulation. Previous studies have demonstrated that the IL-6/STAT3 and JAK2/STAT3 pathways can promote glycolysis, confirming the crucial role of STAT3 in AS and glycolysis $(96,97)$. Therefore, STAT3 was proven to be an important factor in AS and glycolysis.

Evaluation of SRC involvement in endothelial cell glycolysis in AS. SRC is an oncogene that regulates intracellular signal transduction, cell proliferation and cell migration (98). SRC phosphorylation and activation also participates in intracellular metabolic processes (99). It was previously demonstrated that the SRC/AKT/LKB1/AMPK $\alpha$ signalling pathway can shift the intracellular metabolic pathway from glycolysis to aerobic metabolism (100). In addition, SRC-related pathways in endothelial cells promote AS by affecting leukocyte adhesion and monocyte transport (101). Thus, SRC is another important target in AS and endothelial cell glycolysis.

Evaluation of MAPK1 involvement in endothelial cell glycolysis in AS. MAPK is an important intracellular enzyme and the upstream signalling transduction molecule of several pathways (102). It was previously demonstrated that the P38/MAPK signalling pathway can aggravate AS, while MAPK1 affects cell adhesion and the immune response to promote AS development (103). However, further research is required to confirm the regulatory role of MAPK1 in AS and endothelial cell glycolysis.

\section{Discussion}

Although some progress has been made in the treatment of AS and its complications, with a consequent improvement in patient survival (1), long-term illness puts pressure on the heart and is associated with a major socioeconomic burden (3). AS is currently considered as a chronic inflammatory disease, and the underlying mechanisms mainly involve oxidative stress, lipid metabolism disorders, endothelial cell damage, inflammation and immune dysfunction $(5,27)$. Eventually, plaque rupture and thrombosis causes acute life-threatening cardiovascular and cerebrovascular diseases (1). Vascular endothelium inflammation and cell dysfunction are considered as the initiating and central events in AS (29). Since glycolysis is the main metabolic pathway in endothelial cells, an in-depth study of the association between endothelial cell glycolysis and AS may further elucidate AS pathophysiology and provide clues for its prevention (11).

Through mining and analysis of endothelial cell glycolysis and AS-related genes, 8 key targets were identified, namely AKT1, IL-6, VEGFA, TP53, STAT3, SRC and MAPK1. These targets were found to directly or indirectly affect the expression of key rate-limiting enzymes in endothelial cell glycolysis. In addition, the pathways obtained by the enrichment of these genes included the immune system pathway, the signal transduction pathway, pathways in cancer, the PI3K/AKT, IL-4, IL-13, HIF-1, NGF and FoxO signalling pathways, the platelet activation, signalling and aggregation pathway, as well as the fluid shear stress and AS pathways. The aforementioned results are based on existing research and correlation prediction (104). At present, research on AS mainly uses genetically modified animal models, such as $\mathrm{ApoE}^{-/-}$and $\mathrm{db} / \mathrm{db}$ mice, and the findings cannot yet be translated into clinical application. Therefore, these targets must be further verified before they reach the clinical stage. The aim of the present review was to 
contribute to the study of AS mechanisms and provide novel suggestions, since future AS therapies should be explored from a new perspective. Advanced science and technology, including high-content technology, single cell transcriptomics, lipometabolic technology and epigenetic modification technology, will hopefully accelerate the search for novel AS treatments.

\section{Acknowledgements}

Not applicable.

\section{Funding}

This review was supported by the National Natural Science Foundation of China (grant no. 81973514), the Central Public-Interest Scientific Institution Basal Research Fund (grant no. 2018PT35030) and the Drug Innovation Major Project (grant no. 2018ZX09711001-009).

\section{Availability of data and materials}

Not applicable.

\section{Authors' contributions}

GS and XS contributed to the conception and design of the study. RW performed the literature search and wrote the manuscript. MW prepared the figures. JY revised the manuscript. All the authors have read and approved the final manuscript.

\section{Ethics approval and consent to participate}

Not applicable.

\section{Patient consent for publication}

Not applicable.

\section{Competing interests}

The authors declare that they have no competing interests.

\section{References}

1. Kobiyama K and Ley K: Atherosclerosis. Circ Res 123: $1118-1120,2018$

2. Spinelli FR, Barone F, Cacciapaglia F, Pecani A and Piga M: Atherosclerosis and autoimmunity. Mediators Inflamm 2018: 6730421, 2018.

3. Lu H and Daugherty A: Atherosclerosis. Arterioscler Thromb Vasc Biol 35: 485-491, 2015.

4. Libby P, Buring JE, Badimon L, Hansson GK, Deanfield J, Bittencourt MS, Tokgözoğlu L and Lewis EF: Atherosclerosis Nat Rev Dis Primers 5: 56, 2019.

5. Bories GFP and Leitinger N: Macrophage metabolism in atherosclerosis. FEBS Lett 591: 3042-3060, 2017.

6. Zhao Y, Qu H, Wang Y, Xiao W, Zhang Y and Shi D: Small rodent models of atherosclerosis. Biomed Pharmacother 129: 110426, 2020.

7. Goonewardena SN, Prevette LE and Desai AA: Metabolomics and atherosclerosis. Curr Atheroscler Rep 12: 267-272, 2010.

8. Sitia S, Tomasoni L, Atzeni F, Ambrosio G, Cordiano C, Catapano A, Tramontana S, Perticone F, Naccarato P, Camici P, et al: From endothelial dysfunction to atherosclerosis. Autoimmun Rev 9: 830-834, 2010.
9. Chen PY, Schwartz MA and Simons M: Endothelial-tomesenchymal transition, vascular inflammation, and atherosclerosis. Front Cardiovasc Med 7: 53, 2020.

10. Gao F, Chen J and Zhu H: A potential strategy for treating atherosclerosis: Improving endothelial function via AMP-activated protein kinase. Sci China Life Sci 61: 1024-1029, 2018.

11. Pircher A, Treps L, Bodrug N and Carmeliet P: Endothelial cell metabolism: A novel player in atherosclerosis? Basic principles and therapeutic opportunities. Atherosclerosis 253: 247-257, 2016.

12. Zhao X, Tan F, Cao X, Cao Z, Li B, Shen Z and Tian Y: PKM2dependent glycolysis promotes the proliferation and migration of vascular smooth muscle cells during atherosclerosis. Acta Biochim Biophys Sin (Shanghai) 52: 9-17, 2020.

13. Ilhan F and Kalkanli ST: Atherosclerosis and the role of immune cells. World J Clin Cases 3: 345-352, 2015.

14. Xiao W, Jia Z, Zhang Q, Wei C, Wang $\mathrm{H}$ and Wu Y: Inflammation and oxidative stress, rather than hypoxia, are predominant factors promoting angiogenesis in the initial phases of atherosclerosis. Mol Med Rep 12: 3315-3322, 2015.

15. Dinh QN, Chrissobolis S, Diep H, Chan CT, Ferens D, Drummond GR and Sobey CG: Advanced atherosclerosis is associated with inflammation, vascular dysfunction and oxidative stress, but not hypertension. Pharmacol Res 116: 70-76, 2017.

16. Yang K, Zhang H, Luo Y, Zhang J, Wang M, Liao P, Cao L, Guo P, Sun G and Sun X: Gypenoside XVII prevents atherosclerosis by attenuating endothelial apoptosis and oxidative stress: Insight into the ER $\alpha$-Mediated PI3K/Akt Pathway. Int J Mol Sci 18: 77, 2017.

17. Feletou M, Cohen RA, Vanhoutte PM and Verbeuren TJ: TP receptors and oxidative stress hand in hand from endothelial dysfunction to atherosclerosis. Adv Pharmacol 60: 85-106, 2010.

18. Armstrong AW, Voyles SV, Armstrong EJ, Fuller EN and Rutledge JC: Angiogenesis and oxidative stress: Common mechanisms linking psoriasis with atherosclerosis. J Dermatol Sci 63: 1-9, 2011.

19. Gupta M, Blumenthal C, Chatterjee S, Bandyopadhyay D, Jain V, Lavie CJ, Virani SS, Ray KK, Aronow WS and Ghosh RK: Novel emerging therapies in atherosclerosis targeting lipid metabolism. Expert Opin Investig Drugs 29: 611-622, 2020.

20. Lee J, Jung S, Kim N, Shin MJ, Ryu DH and Hwang GS: Myocardial metabolic alterations in mice with diet-induced atherosclerosis: Linking sulfur amino acid and lipid metabolism. Sci Rep 7: 13597, 2017.

21. Novák J, Olejníčková V, Tkáčová N and Santulli G: Mechanistic role of MicroRNAs in coupling lipid metabolism and atherosclerosis. Adv Exp Med Biol 887: 79-100, 2015.

22. Shao D, Lian Z, Di Y, Zhang L, Rajoka MSR, Zhang Y, Kong J, Jiang C and Shi J: Dietary compounds have potential in controlling atherosclerosis by modulating macrophage cholesterol metabolism and inflammation via miRNA. NPJ Sci Food 2: 13, 2018.

23. Giral H, Kratzer A and Landmesser U: MicroRNAs in lipid metabolism and atherosclerosis. Best Pract Res Clin Endocrinol Metab 30: 665-676, 2016.

24. Gimbrone MA Jr and García-Cardeña G: Endothelial cell dysfunction and the pathobiology of atherosclerosis. Circ Res 118: 620-636, 2016.

25. Mudau M, Genis A, Lochner A and Strijdom H: Endothelial dysfunction: The early predictor of atherosclerosis. Cardiovasc J Afr 23: 222-231, 2012.

26. Brix B, Mesters JR, Pellerin L and Johren O: Endothelial cell-derived nitric oxide enhances aerobic glycolysis in astrocytes via HIF-1 $\alpha$-mediated target gene activation. J Neurosci 32: 9727-9735, 2012.

27. Legein B, Temmerman L, Biessen EA and Lutgens E: Inflammation and immune system interactions in atherosclerosis. Cell Mol Life Sci 70: 3847-3869, 2013.

28. Libby $\mathrm{P}$ and Hansson GK: Taming immune and inflammatory responses to treat atherosclerosis. J Am Coll Cardiol 71: 173-176, 2018.

29. Wolf D and Ley K: Immunity and Inflammation in atherosclerosis. Circ Res 124: 315-327, 2019.

30. Hansson GK and Hermansson A: The immune system in atherosclerosis. Nat Immunol 12: 204-212, 2011

31. Iwata $\mathrm{H}$ and Nagai R: Novel immune signals and atherosclerosis. Curr Atheroscler Rep 14: 484-490, 2012.

32. Kuosmanen SM, Kansanen E, Kaikkonen MU, Sihvola V, Pulkkinen K, Jyrkkänen HK, Tuoresmäki P, Hartikainen J, Hippeläinen $\mathrm{M}$, Kokki $\mathrm{H}$, et al: NRF2 regulates endothelial glycolysis and proliferation with miR-93 and mediates the effects of oxidized phospholipids on endothelial activation. Nucleic Acids Res 46: 1124-1138, 2018. 
33. Ouimet M, Ediriweera HN, Gundra UM, Sheedy FJ, Ramkhelawon B, Hutchison SB, Rinehold K, van Solingen C, Fullerton MD, Cecchini K, et al: MicroRNA-33-dependent regulation of macrophage metabolism directs immune cell polarization in atherosclerosis. J Clin Invest 125: 4334-4348, 2015.

34. Kumar S, Kim CW, Simmons RD and Jo H: Role of flow-sensitive microRNAs in endothelial dysfunction and atherosclerosis: Mechanosensitive athero-miRs. Arterioscler Thromb Vasc Biol 34: 2206-2216, 2014

35. Barton M: Mechanisms and therapy of atherosclerosis and its clinical complications. Curr Opin Pharmacol 13: 149-153, 2013.

36. Gonzalez L and Trigatti BL: Macrophage apoptosis and necrotic core development in atherosclerosis: A rapidly advancing field with clinical relevance to imaging and therapy. Can J Cardiol 33: 303-312, 2017

37. Chen C, Wang Y, Cao Y, Wang Q, Anwaier G, Zhang Q and Qi R: Mechanisms underlying the inhibitory effects of probucol on elastase-induced abdominal aortic aneurysm in mice. $\mathrm{Br} J$ Pharmacol 177: 204-216, 2020

38. Guo X, Wang L, Xia X, Wang P and Li X: Effects of atorvastatin and/or probucol on recovery of atherosclerosis in high-fat-diet-fed apolipoprotein E-deficient mice. Biomed Pharmacother 109: 1445-1453, 2019

39. Profumo E, Buttari B, D'Arcangelo D, Tinaburri L, Dettori MA, Fabbri D, Delogu G and Riganò R: The nutraceutical dehydrozingerone and its dimer counteract inflammation- and oxidative stress-induced dysfunction of in vitro cultured human endothelial cells: A novel perspective for the prevention and therapy of atherosclerosis. Oxid Med Cell Longev 2016: 1246485, 2016

40. Jiang Y, Jin M, Chen J, Yan J, Liu P, Yao M, Cai W and Pi R: Discovery of a novel niacin-lipoic acid dimer N2L attenuating atherosclerosis and dyslipidemia with non-flushing effects. Eur J Pharmacol 868: 172871, 2020.

41. Ren Y, Qiao W, Fu D, Han Z, Liu W, Ye W and Liu Z: Traditional Chinese medicine protects against cytokine production as the potential immunosuppressive agents in atherosclerosis. J Immunol Res 2017: 7424307, 2017.

42. Li TT, Wang ZB, Li Y, Cao F, Yang BY and Kuang HX: The mechanisms of traditional Chinese medicine underlying the prevention and treatment of atherosclerosis. Chin J Nat Med 17: 401-412, 2019

43. Tian F, Gu L, Si A, Yao Q,Zhang X,Zhao J and Hu D: Metabolomic study on the faecal extracts of atherosclerosis mice and its application in a traditional Chinese Medicine. J Chromatogr B Analyt Technol Biomed Life Sci 1007: 140-148, 2015.

44. Fan Q, Liu Y, Rao J, Zhang Z, Xiao W, Zhu T, Chai X, Ye K, Ning N, Yin Z, et al: Anti-atherosclerosis effect of angong niuhuang pill via regulating Th17/Treg immune balance and inhibiting chronic inflammatory on $\mathrm{ApoE}^{-/-}$mice model of early and mid-term atherosclerosis. Front Pharmacol 10: 1584, 2020.

45. Zhu ZB, Song K, Huang WJ, Li H, Yang H, Bai YQ, Guo KT, Yang RB, Lou WJ, Xia CH, et al: Si-Miao-Yong-An (SMYA) decoction may protect the renal function through regulating the autophagy-mediated degradation of ubiquitinated protein in an atherosclerosis model. Front Pharmacol 11: 837, 2020

46. Li L, Yu AL, Wang ZL, Chen K, Zheng W, Zhou JJ, Xie Q, Yan HB Ren $P$ and Huang X: Chaihu-Shugan-San and absorbed meranzin hydrate induce anti-atherosclerosis and behavioral improvements in high-fat diet $\mathrm{ApoE}^{-/-}$mice via anti-inflammatory and BDNF-TrkB pathway. Biomed Pharmacother 115: 108893, 2019.

47. Haskard DO, Boyle JJ, Evans PC, Mason JC and Randi AM Cytoprotective signaling and gene expression in endothelial cells and macrophages-lessons for atherosclerosis. Microcirculation 20: 203-216, 2013.

48. Dong Y, Fernandes C, Liu Y, Wu Y, Wu H, Brophy ML, Deng L, Song K, Wen A, Wong S, et al: Role of endoplasmic reticulum stress signalling in diabetic endothelial dysfunction and atherosclerosis. Diab Vasc Dis Res 14: 14-23, 2017.

49. Chrysohoou C, Kollia N and Tousoulis D: The link between depression and atherosclerosis through the pathways of inflammation and endothelium dysfunction. Maturitas 109: 1-5, 2018.

50. Jensen HA and Mehta JL: Endothelial cell dysfunction as a novel therapeutic target in atherosclerosis. Expert Rev Cardiovasc Ther 14: 1021-1033, 2016

51. Theodorou K and Boon RA: Endothelial cell metabolism in atherosclerosis. Front Cell Dev Biol 6: 82, 2018

52. Verma I, Syngle A, Krishan P and Garg N: Endothelial progenitor cells as a marker of endothelial dysfunction and atherosclerosis in Ankylosing Spondylitis: A cross-sectional study. Int J Angiol 26: $36-42,2017$.
53. Lee DY and Chiu JJ: Atherosclerosis and flow: Roles of epigenetic modulation in vascular endothelium. J Biomed Sci 26: 56, 2019.

54. Georgescu A, Alexandru N, Andrei E, Dragan E, Cochior D and Dias S: Effects of transplanted circulating endothelial progenitor cells and platelet microparticles in atherosclerosis development Biol Cell 108: 219-243, 2016.

55. Xiang W, Hu ZL, He XJ and Dang XQ: Intravenous transfusion of endothelial progenitor cells that overexpress vitamin D receptor inhibits atherosclerosis in apoE-deficient mice. Biomed Pharmacother 84: 1233-1242, 2016.

56. Kong M, Zhao Y, Chen A and Lin A: The importance of physiologic ischemia training in preventing the development of atherosclerosis: The role of endothelial progenitor cells in atherosclerotic rabbits. Coron Artery Dis 30: 377-383, 2019.

57. Pákozdi A, Besenyei T, Paragh G, Koch AE and Szekanecz Z: Endothelial progenitor cells in arthritis-associated vasculogenesis and atherosclerosis. Joint Bone Spine 76: 581-583, 2009.

58. Hu X, Cai X, Ma R, Fu W, Zhang C and Du X: Iron-load exacerbates the severity of atherosclerosis via inducing inflammation and enhancing the glycolysis in macrophages. J Cell Physiol 234: 18792-18800, 2019.

59. Flynn MC, Kraakman MJ, Tikellis C, Lee MKS, Hanssen NMJ, Kammoun HL, Pickering RJ, Dragoljevic D, Al-Sharea A, Barrett TJ, et al: Transient intermittent hyperglycemia accelerates atherosclerosis by promoting myelopoiesis. Circ Res 127 : 877-892, 2020.

60. Matsuura Y, Yamashita A, Zhao Y, Iwakiri T, Yamasaki K, Sugita C, Koshimoto C, Kitamura K, Kawai K, Tamaki N, et al: Altered glucose metabolism and hypoxic response in alloxaninduced diabetic atherosclerosis in rabbits. PLoS One 12: e0175976, 2017.

61. Vojinovic D, van der Lee SJ, van Duijn CM, Vernooij MW, Kavousi M, Amin N, Demirkan A, Ikram MA, van der Lugt A and Bos D: Metabolic profiling of intra- and extracranial carotid artery atherosclerosis. Atherosclerosis 272: 60-65, 2018.

62. Akins NS, Nielson TC and Le HV: Inhibition of glycolysis and glutaminolysis: An emerging drug discovery approach to combat cancer. Curr Top Med Chem 18: 494-504, 2018.

63. Deng F, Zhou R, Lin C, Yang S, Wang H, Li W, Zheng K, Lin W, Li X, Yao X, et al: Tumor-secreted dickkopf2 accelerates aerobic glycolysis and promotes angiogenesis in colorectal cancer. Theranostics 9: 1001-1014, 2019.

64. Godfrey R and Quinlivan R: Skeletal muscle disorders of glycogenolysis and glycolysis. Nat Rev Neurol 12: 393-402, 2016

65. Schoors S, De Bock K, Cantelmo AR, Georgiadou M, Ghesquière B, Cauwenberghs S, Kuchnio A, Wong BW, Quaegebeur A, Goveia J, et al: Partial and transient reduction of glycolysis by PFKFB3 blockade reduces pathological angiogenesis. Cell Metab 19: 37-48, 2014.

66. Fernie AR, Carrari F and Sweetlove LJ: Respiratory metabolism: Glycolysis, the TCA cycle and mitochondrial electron transport. Curr Opin Plant Biol 7: 254-261, 2004.

67. Tang BL: Glucose, glycolysis, and neurodegenerative diseases. J Cell Physiol 235: 7653-7662, 2020.

68. Paik JY, Lee KH, Ko BH, Choe YS, Choi Y and Kim BT: Nitric oxide stimulates 18F-FDG uptake in human endothelial cells through increased hexokinase activity and GLUT1 expression. J Nucl Med 46: 365-370, 2005.

69. Kim JW, Gao P, Liu YC, Semenza GL and Dang CV: Hypoxia-inducible factor 1 and dysregulated c-Myc cooperatively induce vascular endothelial growth factor and metabolic switches hexokinase 2 and pyruvate dehydrogenase kinase 1. Mol Cell Biol 27: 7381-7393, 2007.

70. Song J, Li Y, Song J, Hou F, Liu B and Li A: Mangiferin protects mitochondrial function by preserving mitochondrial hexokinase-II in vessel endothelial cells. Biochim Biophys Acta Mol Basis Dis 1863: 1829-1839, 2017.

71. Wang Y, Han X, Fu M, Wang J, Song Y, Liu Y, Zhang J, Zhou J and Ge J: Qiliqiangxin attenuates hypoxia-induced injury in primary rat cardiac microvascular endothelial cells via promoting HIF-1 $\alpha$-dependent glycolysis. J Cell Mol Med 22: 2791-2803, 2018

72. Wik JA, Lundbäck P, la Cour Poulsen L, Haraldsen G, Skålhegg BS and Hol J: 3PO inhibits inflammatory NFkB and stress-activated kinase signaling in primary human endothelial cells independently of its target PFKFB3. PLoS One 15: e0229395, 2020.

73. Zhang R, Li R, Liu Y, Li L and Tang Y: The glycolytic enzyme PFKFB3 controls TNF- $\alpha$-induced endothelial proinflammatory responses. Inflammation 42: 146-155, 2019. 
74. Lu S, Deng J, Liu H, Liu B, Yang J, Miao Y, Li J, Wang N, Jiang C, $\mathrm{Xu} \mathrm{Q}$, et al: PKM2-dependent metabolic reprogramming in $\mathrm{CD}^{+} \mathrm{T}$ cells is crucial for hyperhomocysteinemia-accelerated atherosclerosis. J Mol Med (Berl) 96: 585-600, 2018.

75. Zhang X, Chen B, Wu J, Sha J, Yang B, Zhu J, Sun J, Hartung J and Bao E: Aspirin enhances the protection of Hsp90 from heat-stressed injury in cardiac microvascular endothelial cells through PI3K-Akt and PKM2 pathways. Cells 9: 243, 2020.

76. Serganova I, Cohen IJ, Vemuri K, Shindo M, Maeda M, Mane M, Moroz E, Khanin R, Satagopan J, Koutcher JA and Blasberg R: LDH-A regulates the tumor microenvironment via HIF-signaling and modulates the immune response. PLoS One 13: e0203965, 2018.

77. Chen SF, Pan MX, Tang JC, Cheng J, Zhao D, Zhang Y, Liao HB, Liu R, Zhuang Y, Zhang ZF, et al: Arginine is neuroprotective through suppressing HIF-1 $\alpha /$ LDHA-mediated inflammatory response after cerebral ischemia/reperfusion injury. Mol Brain 13: 63, 2020.

78. Fernández-Hernando C, József L, Jenkins D, Di Lorenzo A and Sessa WC: Absence of Akt1 reduces vascular smooth muscle cell migration and survival and induces features of plaque vulnerability and cardiac dysfunction during atherosclerosis. Arterioscler Thromb Vasc Biol 29: 2033-2040, 2009.

79. Pan C, Liu Q and Wu X: HIF1 $\alpha /$ miR-520a-3p/AKT1/mTOR feedback promotes the proliferation and glycolysis of gastric cancer cells. Cancer Manag Res 11: 10145-10156, 2019.

80. Zhong ZW, Zhou WC, Sun XF, Wu QC, Chen WK and Miao CH: Dezocine regulates the malignant potential and aerobic glycolysis of liver cancer targeting Akt1/GSK-3 $\beta$ pathway. Ann Transl Med 8: 480, 2020

81. Zhao X, Wu X, Wang H, Yu H and Wang J: USP53 promotes apoptosis and inhibits glycolysis in lung adenocarcinoma through FKBP51-AKT1 signaling. Mol Carcinog 59: 1000-1011, 2020.

82. Song L and Schindler C: IL-6 and the acute phase response in murine atherosclerosis. Atherosclerosis 177: 43-51, 2004.

83. Zhang P, Chen X, Zhang Y, Su H, Zhang Y, Zhou X, Sun M, Li L and $\mathrm{Xu} Z$ : Tet 3 enhances IL-6 expression through up-regulation of 5-hmC in IL-6 promoter in chronic hypoxia induced atherosclerosis in offspring rats. Life Sci 232: 116601, 2019

84. Bozic M, Alvarez A, de Pablo C, Sanchez-Niño MD, Ortiz A Dolcet X, Encinas M, Fernandez E and Valdivielso JM: Impaired vitamin D signaling in endothelial cell leads to an enhanced leukocyte-endothelium interplay: Implications for atherosclerosis development. PLoS One 10: e0136863, 2015.

85. Han J, Meng Q, Xi Q, Zhang Y, Zhuang Q, Han Y, Jiang Y, Ding $Q$ and Wu G: Interleukin-6 stimulates aerobic glycolysis by regulating PFKFB3 at early stage of colorectal cancer. Int J Oncol 48: 215-224, 2016.

86. Li H, Liang Q and Wang L: Icaritin inhibits glioblastoma cell viability and glycolysis by blocking the IL-6/Stat 3 pathway. J Cell Biochem, Nov 2, 2018 (Epub ahead of print). doi: 10.1002/jcb.28000.

87. Zhao $\mathrm{N}$ and Zhang J: Role of alternative splicing of VEGF-A in the development of atherosclerosis. Aging (Albany NY) 10 2695-2708, 2018.

88. Wang X, Hu Z, Wang Z, Cui Y and Cui X: Angiopoietin-like protein 2 is an important facilitator of tumor proliferation, metastasis, angiogenesis and glycolysis in osteosarcoma. Am J Trans Res 11: 6341-6355, 2019.

89. Peek CB, Levine DC, Cedernaes J, Taguchi A, Kobayashi Y, Tsai SJ, Bonar NA, McNulty MR, Ramsey KM and Bass J: Circadian clock interaction with HIF1 $\alpha$ mediates oxygenic metabolism and anaerobic glycolysis in skeletal muscle. Cell Metab 25: 86-92, 2017.

90. Zhao M, Fan J, Liu Y, Yu Y, Xu J, Wen Q, Zhang J, Fu S, Wang B, Xiang L, et al: Oncogenic role of the TP53-induced glycolysis and apoptosis regulator in nasopharyngeal carcinoma through $\mathrm{NF}-\kappa \mathrm{B}$ pathway modulation. Int J Oncol 48: 756-764, 2016.
91. KoYH,Domingo-Vidal M,RocheM,LinZ,Whitaker-MenezesD, Seifert E, Capparelli C, Tuluc M, Birbe RC, Tassone P, et al: TP53-inducible glycolysis and apoptosis regulator (TIGAR) metabolically reprograms carcinoma and stromal cells in breast cancer. J Biol Chem 291: 26291-26303, 2016.

92.Xiong Y, Yepuri G, Forbiteh M, Yu Y, Montani JP, Yang Z and Ming XF: ARG2 impairs endothelial autophagy through regulation of MTOR and PRKAA/AMPK signaling in advanced atherosclerosis. Autophagy 10: 2223-2238, 2014.

93. Li X, Wu L, Zopp M, Kopelov S and Du W: p53-TP53-Induced glycolysis regulator mediated glycolytic suppression attenuates DNA damage and genomic instability in fanconi anemia hematopoietic stem cells. Stem Cells 37: 937-947, 2019.

94. Chen Q, Lv J, Yang W, Xu B, Wang Z, Yu Z, Wu J, Yang Y and Han Y: Targeted inhibition of STAT3 as a potential treatment strategy for atherosclerosis. Theranostics 9: 6424-6442, 2019.

95. Zhou X, Li D, Yan W and Li W: Pravastatin prevents aortic atherosclerosis via modulation of signal transduction and activation of transcription 3 (STAT3) to attenuate interleukin-6 (IL-6) action in ApoE knockout mice. Int J Mol Sci 9: 2253-2264, 2008

96. Li Y, Wang Y, Liu Z, Guo X, Miao Z and Ma S: Atractylenolide I induces apoptosis and suppresses glycolysis by blocking the JAK2/STAT3 signaling pathway in colorectal cancer cells. Front Pharmacol 11: 273, 2020.

97. Zheng M, Cao MX, Yu XH, Li L, Wang K, Wang SS, Wang HF, Tang YJ, Tang YL and Liang XH: STAT3 promotes invasion and aerobic glycolysis of human oral squamous cell carcinoma via inhibiting FoxO1. Front Oncol 9: 1175, 2019.

98. Seki N, Hashimoto N, Taira M, Yagi S, Yoshida Y, Ishikawa K, Suzuki Y, Sano H, Horiuchi S, Yoshida S, et al: Regulation of Src homology 2-containing protein tyrosine phosphatase by advanced glycation end products: The role on atherosclerosis in diabetes. Metabolism 56: 1591-1598, 2007.

99. Park JS, Lee S, Jeong AL, Han S, Ka HI, Lim JS, Lee MS, Yoon DY, Lee JH and Yang Y: Hypoxia-induced IL-32 increases glycolysis in breast cancer cells. Cancer Lett 356: $800-808,2015$.

100. Nam K, Oh S and Shin I: Ablation of CD44 induces glycolysis-to-oxidative phosphorylation transition via modulation of the c-Src-Akt-LKB1-AMPK $\alpha$ pathway. Biochem J 473: 3013-3030, 2016.

101. Byun S, Jung H, Chen J, Kim YC, Kim DH, Kong B, Guo G, Kemper B and Kemper JK: Phosphorylation of hepatic farnesoid X receptor by FGF19 signaling-activated Src maintains cholesterol levels and protects from atherosclerosis. J Biol Chem 294 8732-8744, 2019.

102. Lovren F, Pan Y, Shukla PC, Quan A, Teoh H, Szmitko PE, Peterson MD, Gupta M, Al-Omran M and Verma S: Visfatin activates eNOS via Akt and MAP kinases and improves endothelial cell function and angiogenesis in vitro and in vivo: Translational implications for atherosclerosis. Am J Physiol Endocrinol Metab 296: 1440-1449, 2009.

103. Gupta A, Mohanty P and Bhatnagar S: Integrative analysis of ocular complications in atherosclerosis unveils pathway convergence and crosstalk. J Recept Signal Transduct Res 35: 149-164, 2015.

104. Perrotta P, Emini Veseli B, Van der Veken B, Roth L, Martinet W and De Meyer GRY: Pharmacological strategies to inhibit intra-plaque angiogenesis in atherosclerosis. Vascul Pharmacol 112: 72-78, 2019.

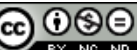

This work is licensed under a Creative Common Attribution-NonCommercial-NoDerivatives 4.0 International (CC BY-NC-ND 4.0) License. 